\title{
Efficacy and Safety of Immunosuppressive Therapy for PBC-AIH Overlap Syndrome Accompanied by Decompensated Cirrhosis: A Real-World Study
}

\author{
Xiaoli Fan, ${ }^{1}$ Yongjun Zhu, ${ }^{1}$ Ruoting Men, ${ }^{1}$ Maoyao Wen, ${ }^{1}$ Yi Shen, ${ }^{1}$ Changli Lu, \\ and Li Yang ${ }^{1}$ \\ ${ }^{1}$ Department of Gastroenterology \& Hepatology, West China Hospital, Sichuan University, Chengdu, Sichuan 610041, China \\ ${ }^{2}$ Department of Pathology, West China Hospital, Sichuan University, Chengdu, Sichuan 610041, China
}

Correspondence should be addressed to Li Yang; yangli_hx@scu.edu.cn

Received 28 January 2018; Revised 2 June 2018; Accepted 9 July 2018; Published 2 August 2018

Academic Editor: Kusum Kharbanda

Copyright (C) 2018 Xiaoli Fan et al. This is an open access article distributed under the Creative Commons Attribution License, which permits unrestricted use, distribution, and reproduction in any medium, provided the original work is properly cited.

Aim. To explore the efficacy and safety of immunosuppressive therapy for the treatment of primary biliary cirrhosis-autoimmune hepatitis (PBC-AIH) overlap syndrome accompanied by decompensated cirrhosis. Methods. A cohort study was performed to evaluate the usefulness of immunosuppressive therapy in this unique group. This cohort study was performed between October 2013 and June 2017 and included 28 biopsy-proven patients diagnosed according to the Paris criteria. The therapies included ursodeoxycholic acid (UDCA) alone ( $\mathrm{N}=14)$ or in combination with immunosuppression (IS) therapy (N=14). The primary endpoints were biochemical remission, liver-related adverse events, transplant-free survival, and drug side-effects. Results. The frequency of biochemical remission for the AIH features was significantly higher in the UDCA+IS group than in the UDCA-only group ( 60.0 versus $9.1 \%, \mathrm{P}=0.024$ ) after 12 months of therapy but not after 3 and 6 months $(28.6$ versus $0 \%, P=0.165 ; 35.7$ versus $7.1 \%$, $\mathrm{P}=0.098)$. The rates of liver-related adverse events were lower in the combined group ( $2 / 14$ versus $9 / 14, \mathrm{P}=0.018)$. The Kaplan-Meier estimate showed that the transplant-free survival was distinct between the two groups $(\mathrm{P}=0.019)$. In the UDCA+IS group, mild and transient leukopenia occurred in two patients receiving azathioprine (AZA), and an infection was observed in one patient receiving mycophenolate mofetil (MMF). Conclusions. PBC-AIH patients with decompensated cirrhosis receiving a combination of UDCA and immunosuppressors presented with higher biochemical remission rates and experienced fewer liver-related adverse events, implying that the combined treatment might be a better therapeutic option for strictly defined decompensated PBC-AIH overlap syndrome.

\section{Introduction}

Autoimmune liver disease (AILD) comprises a group of immune-mediated liver diseases that include autoimmune hepatitis (AIH), primary biliary cirrhosis (PBC), and primary sclerosing cholangitis (PSC) [1]. The occurrence of overlapping syndromes at different disease stages (so-called overlap syndromes) is not rare, with PBC-AIH overlap syndrome being the most common [2]. The prevalence of PBC-AIH overlap syndrome is approximately $8-10 \%$ in adult patients with either $\mathrm{PBC}$ or $\mathrm{AIH}[3,4]$, and this low incidence contributes to imprecise diagnostic criteria; furthermore, no standard therapy is currently available. According to the most recent guidelines based on the results of small studies, a combination of ursodeoxycholic acid (UDCA) and immunosuppressants is often recommended for PBC-AIH in clinical practice [5-7].

Risk stratification was recently performed by a panel of experts on the topic of cirrhosis [8]. The survival rates of compensated and decompensated cirrhosis are strikingly different, and the median survival time of the latter may be less than 2 years $[9,10]$. PBC-AIH patients presenting with already advanced cirrhosis may die in the early phase of treatment because of complications related to immunosuppression; however, there are no data supporting this finding [11]. Hence, the present study was undertaken to 
analyse a single-centre cohort of PBC-AIH patients with decompensated cirrhosis. The present study was initiated to determine whether immunosuppressive therapy could be used in a cohort of decompensated PBC-AIH patients to obtain a biochemical response and to control the disease progression and the cost in terms of adverse reactions.

\section{Materials and Methods}

2.1. Inclusion Criteria. West China Hospital is a 4300-bed tertiary teaching hospital affiliated with Sichuan University. The hospital has a liver transplant unit (Liver Transplantation Centre, West China Hospital) and is the leading hospital in the western areas of China.

Cases were prospectively recruited between October 2013 and June 2017 using an electronic database of AILD, which was established in October 2013. Twenty-eight consecutive patients with PBC-AIH with decompensated cirrhosis strictly according to the Paris criteria were incorporated. The study was approved by the Ethics Committee of West China Hospital.

As recommended by the Paris criteria, the patients were diagnosed with $\mathrm{PBC}$ when they met 2 or more of the following diagnostic criteria: (1) the presence of anti-mitochondrial antibodies (AMA), (2) an alkaline phosphatase (ALP) level at least 2-fold the upper normal limit (UNL) or a gammaglutamyl transpeptidase (GGT) level at least 5-fold the UNL, and (3) a liver biopsy specimen exhibiting florid bile duct lesions. Patients were diagnosed with AIH when they met 2 or more of the following criteria: (1) an alanine aminotransferase (ALT) level at least 5-fold the UNL, (2) serum IgG at least 2-fold the UNL or a positive test for smooth muscle antibodies (SMA), and (3) a liver biopsy exhibiting moderate or severe periportal or periseptal lymphocytic piecemeal necrosis. In our setting, only simultaneous forms of PBCAIH overlap syndrome were enrolled, because fewer patients had the consecutive forms, which might have raised different diagnostic and therapeutic issues $[12,13]$. Patients with viral hepatitis, nonalcoholic steatohepatitis, drug-induced liver disease, Wilson's disease, or other causes of liver damage were excluded through a careful history analysis and evaluation. Overlap with suspected PSC and acute severe AIH, as defined according to the proposed criteria, was also excluded [14].

Cirrhosis was diagnosed according to the histological analysis, unequivocal imaging, or endoscopic examination [15]. Decompensation was diagnosed by the presence of clinical complications, including ascites, variceal hemorrhage, and hepatic encephalopathy (HE) [8].

2.2. Clinical and Laboratory Analyses. The biochemical, serological, radiological, and histological data, treatment strategies, and outcomes were recorded for the patients with PBC-AIH with decompensated cirrhosis. The laboratory measurements included total bilirubin (TBIL), ALT, aspartate aminotransferase (AST), ALP, GGT, albumin (ALB), globulin (GLB), IgG, IgM, antinuclear antibody (ANA), liver-kidney microsomal antibody (LKM), soluble liver antigen (SLA), antibody against liver cytosol type 1 antigen (LC-1), routine blood measurements, and noninvasive hepatic fibrosis parameters. Child-Pugh scores were collected at baseline. All parameters were examined in the Department of Laboratory Medicine of West China Hospital, which was certified by the College of American Pathologists (CAP).

The imaging tests included ultrasonography (US), computed tomography (CT), and/or magnetic resonance imaging (MRI).

All patients underwent follow-up, including clinical and laboratory evaluations, every 1-3 months.

2.3. Treatment. A total of 28 consecutive decompensated PBC-AIH patients were enrolled in this open, real-world, observational study. Because the optimal type of treatment was not known, the managing physician was free to decide whether he would treat the patients with UDCA alone or combined with immunosuppressants. The patients treated with UDCA alone received a $13-15 \mathrm{mg} / \mathrm{kg} / \mathrm{d}$ dose. In the UDCA+IS group $(\mathrm{N}=14)$, the patients were given an initial dose of $12-40 \mathrm{mg} / \mathrm{d}$ of methylprednisolone, simultaneously. Induction therapy was response-guided and individualized for the 14 patients and was gradually reduced. Azathioprine (AZA) $(50-100 \mathrm{mg} / \mathrm{d})$ was combined with UDCA and steroids for 12 patients in the UDCA+IS group, whereas mycophenolate mofetil (MMF) was combined with UDCA and steroids for one patient when the total bilirubin level was above 100 $\mu \mathrm{mol} / \mathrm{L}$ and the other one was merely given UDCA and steroids. Proton pump inhibitors (PPIs) were used to prevent peptic ulcers or bleeding during corticosteroid reduction, and vitamin $\mathrm{D}$ and calcium were introduced to prevent osteoporosis and fractures.

Prior to the initiation of corticosteroid therapy, an absence of infection was confirmed by negative cultures of blood samples, ascites fluids, and urine specimens and chest $\mathrm{X}$-ray or CT. Common complications were prevented and treated according to accepted clinical management guidelines.

2.4. Pathological Examination. Liver samples were acquired by ultrasound-guided percutaneous needle biopsy of the liver. The tissues were fixed in 10\% formaldehyde (Kelong, China), embedded in paraffin, and used for haematoxylin and eosin (H\&E) staining, Masson trichrome staining, special staining, and immunohistochemical staining to examine the histological characteristics. Finally, two pathologists (Changli Lu and Jianping Liu) from the Pathology Department of West China Hospital (certified by CAP) interpreted the samples. Diagnostic pathological changes in AIH were recorded, including interface hepatitis, lymphoplasmacytic infiltrate, hepatocyte resetting, and emperipolesis. Pathological changes used for the diagnosis of $\mathrm{PBC}$ included florid duct lesion, bile duct damage, ductular proliferation, and cholestasis. The activity grade (G0-4) and fibrosis stage ( $\mathrm{S} 0-4)$ were assessed, according to the Scheuer system [16-19].

2.5. Outcomes Assessment. The primary effectiveness assessment was biochemical remission of AIH features (normalization of transaminases and IgG after starting therapy, as determined using existing guidelines). Liver-related adverse events 
TABLE 1: Demographic and clinical features, laboratory parameters, prognostic scores, and decompensation characteristics between the two groups.

\begin{tabular}{|c|c|c|c|}
\hline Variables & UDCA-only group $(\mathrm{N}=14)$ & UDCA+IS group $(\mathrm{N}=14)$ & $P$ value \\
\hline Age at entry (years) & $60.0(51.3,61.3)$ & $48.0(42.5,53.5)$ & 0.024 \\
\hline Gender (F/M) & $13 / 1$ & $12 / 2$ & $>0.999$ \\
\hline $\mathrm{TBIL}, \mu \mathrm{mol} / \mathrm{L}$ & $29.0(23.2,41.1)$ & $38.9(35.2,127.1)$ & 0.035 \\
\hline ALT, IU/L & $66.5(44.8,127.0)$ & $112.0(45.7,174.5)$ & 0.401 \\
\hline AST, IU/L & $103.5(78.5,129.8)$ & $170.0(91.0,212.5)$ & 0.401 \\
\hline ALP, IU/L & $349.5(257.8,519.0)$ & $294.0(196.5,430.5)$ & 0.285 \\
\hline GGT, IU/L & $268.5(157.0,656.3)$ & $229.5(51.8,293.5)$ & 0.285 \\
\hline $\mathrm{ALB}, \mathrm{g} / \mathrm{L}$ & $37.6(31.8,39.9)$ & $32.3(29.9,35.5)$ & 0.031 \\
\hline GLB, g/L & $43.4(37.4,47.1)$ & $45.4(35.3,50.2)$ & 0.511 \\
\hline INR & $1.1(1.0,1.2)$ & $1.1(1.0,1.2)$ & 0.734 \\
\hline $\mathrm{Cr}, \mu \mathrm{mol} / \mathrm{L}$ & $57.3(51.8,66.3)$ & $53.5(43.3,61.3)$ & 0.137 \\
\hline ANA positive, $\mathrm{N}(\%)$ & $13 / 14$ & $12 / 14$ & $>0.999$ \\
\hline AMA positive, $\mathrm{N}(\%)$ & $9 / 14$ & $9 / 14$ & $>0.999$ \\
\hline LKM positive, $\mathrm{N}(\%)$ & 0 & 0 & - \\
\hline LC-1 positive, $\mathrm{N}(\%)$ & 0 & 0 & - \\
\hline SLA positive, $\mathrm{N}(\%)$ & 0 & 0 & - \\
\hline Concurrent autoimmune diseases, $\mathrm{N}(\%)$ & $4 / 14$ & $6 / 14$ & 0.695 \\
\hline $\operatorname{IgG}(g / L)$ & $22.8(20.2,25.7)$ & $28.6(19.7,32.4)$ & 0.125 \\
\hline $\operatorname{IgM}(\mathrm{g} / \mathrm{L})$ & $2.2(1.3,5.7)$ & $2.7(2.4,3.0)$ & 0.306 \\
\hline APRI score & $3.9(2.2,5.3)$ & $6.4(3.9,7.2)$ & 0.051 \\
\hline FIB-4 index & $6.6(4.9,8.7)$ & $11.7(6.7,18.9)$ & 0.075 \\
\hline \multicolumn{4}{|l|}{ Complications } \\
\hline Ascites & $12 / 14$ & $10 / 14$ & 0.648 \\
\hline Variceal bleeding & $2 / 14$ & $4 / 14$ & 0.648 \\
\hline \multicolumn{4}{|l|}{ Prognostic scores } \\
\hline Child-Pugh score & $6.5(6.0,7.0)$ & $8.0(6.0,8.0)$ & 0.265 \\
\hline
\end{tabular}

Note. UDCA, ursodeoxycholic acid; IS, immunosuppressants; TBIL, total bilirubin; ALT, alanine aminotransferase; AST, aspartate aminotransferase; ALP, alkaline phosphatase; GGT, gamma-glutamyl transpeptidase; ALB, albumin; GLB, globulin; ANA, antinuclear antibody; AMA, anti-mitochondrial antibody; LKM, liver-kidney microsomal antibody; LC-1, antibody against liver cytosol type 1 antigen; SLA, soluble liver antigen/liver pancreas antibody; APRI, aspartate aminotransferase to platelet ratio index; FIB-4, fibrosis 4 index.

were determined if the patients experienced the following: (1) worsening of existing hepatic decompensation, (2) a new decompensation event other than the event present at the initiation of therapy, (3) liver failure, or (4) transplantation or death attributable to decompensation events. The transplantfree survival was compared additionally. The evaluation of drug side-effects included diarrhoea, steroid-specific side effects (i.e., osteoporosis, fractures, moon face, or acne), infection, and myelosuppression. All safety assessments were performed for the total patient cohort. Patients were followed until May 2018.

2.6. Statistical Analysis. Continuous data are presented as medians and quartiles, and categorical data are expressed as percentages. The Mann-Whitney $U$ test was used for comparisons of continuous variables. The Chi-square test or Fisher's exact test was used to analyse differences in categorical variables between two independent groups where appropriate. A P-value $<0.05$ was considered significant. Data processing was performed with the SPSS software package
(SPSS version 24.0 for Windows, IBM Corp., Armonk, NY, USA).

\section{Results}

3.1. Basic Information. A total of 28 patients diagnosed with PBC-AIH accompanied by decompensated cirrhosis were enrolled in the cohort study (Figure 1). Table 1 presents the clinical and biochemical characteristics of the two groups. For the UDCA alone group, the median age at treatment initiation was $60.0(51.3,61.3)$ years, and the female-to-male ratio was 13:1. For the UDCA+IS group, the median age was $48.0(42.5,53.5)$ years, and the female-to-male ratio was 12:2. AMA was positive in $64.2 \%$ of patients ( 9 of 14 ) in the both the UDCA alone group and UDCA+IS group $(\mathrm{P}>0.999)$. SLA, LC-1, and LKM were negative in the enrolled patients. At the time of study inclusion, the patients who did not receive immunosuppressants had lower total bilirubin (TBIL) levels (29.0 versus $38.9 \mathrm{IU} / \mathrm{L}, \mathrm{P}=0.035)$ and higher albumin $(\mathrm{ALB})$ levels (37.6 versus $32.3 \mathrm{IU} / \mathrm{L}, \mathrm{P}=0.031$ ) than the combined 


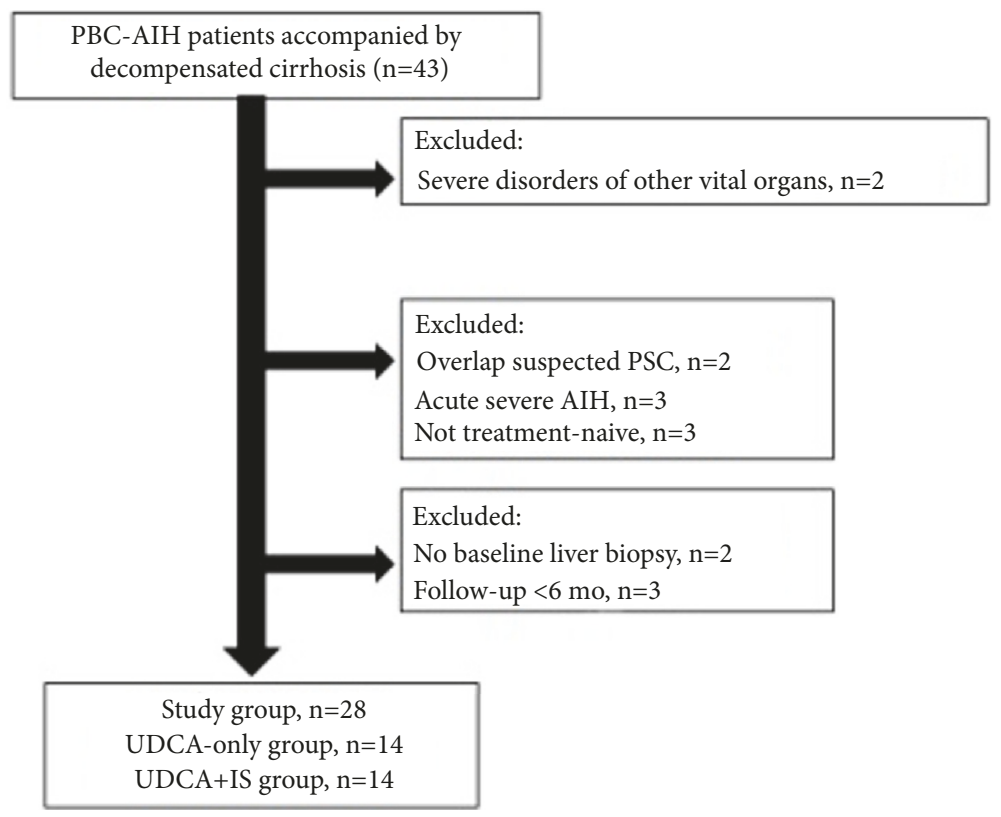

FIGURE 1: Study flowchart for patient inclusion.

TABLE 2: Comparison of symptoms between the two groups at baseline.

\begin{tabular}{lccc}
\hline Symptoms & UDCA-only group (N=14) & UDCA+IS group (N=14) & P value \\
\hline Jaundice & $3(21.4 \%)$ & $6(42.9 \%)$ & 0.420 \\
Ventosity & $5(35.7 \%)$ & $3(21.4 \%)$ & 0.678 \\
Fatigue & $4(28.6 \%)$ & $3(21.4 \%)$ & $>0.999$ \\
Lower limb swelling & $3(21.4 \%)$ & $4(28.6 \%)$ & $>0.999$ \\
Anorexia & $2(14.3 \%)$ & $3(21.4 \%)$ & $>0.999$ \\
Arthralgia & $2(14.3 \%)$ & $4(28.6 \%)$ & 0.648 \\
Yellow urine & $2(14.3 \%)$ & $3(21.4 \%)$ & $>0.999$ \\
Abdominal pain & $3(21.4 \%)$ & $2(14.3 \%)$ & $>0.999$ \\
Weight loss & $2(14.3 \%)$ & $1(7.2 \%)$ & $>0.999$ \\
Nausea & $1(7.2 \%)$ & $1(7.2 \%)$ & - \\
None & $1(7.2 \%)$ & $1(7.2 \%)$ & $>0.999$ \\
\hline
\end{tabular}

group. All patients were diagnosed with decompensated cirrhosis prior to treatment (Table 1).

The common clinical symptoms are shown in Table 2. Jaundice and ventosity were the two most common symptoms in all patients. The symptoms at presentation were similar in the two groups.

3.2. Histological Features in the Study Cohort. All 28 patients had biopsy specimens available at diagnosis and presented with a typical picture of AIH features, with moderate to severe interface hepatitis and lymphocytic infiltrates, as well as PBC features. Severe interface hepatitis was only observed in 2 patients in the combined group. Hepatocellular rosette formation was noted in 8 and 10 patients in the two groups, respectively. Lymphoplasmacytic infiltrate was found in most patients, whereas emperipolesis was not observed. Bile duct damage was found in 13 and 10 patients in the two groups, whereas ductopenia was observed in 9 and 6 patients, respectively. Ductular proliferation, which was revealed by staining for cytokeratin 7, was observed in 11 and 9 patients in the two groups, respectively (Table 3). Figure 2 presents the features of a biopsy specimen from a patient who received UDCA plus immunosuppressant treatment.

3.3. Treatment Response. Table 4 shows the treatment response rates for both patient groups. At the end of the study, $42.9 \%(12 / 28)$ of the patients reached complete biochemical remission. Overall, the response rates after 3,6 , and 12 months of therapy were $14.2 \%(4 / 28), 21.4 \%(6 / 28)$, and $33.3 \%$ (7/28), respectively. The remission rate after 12 months was significantly higher in the combined group than in the UDCA-only group (60.0 versus $9.1 \%, \mathrm{P}=0.024)$. Although not statistically significant, the biochemical remission rates seem to be higher in the combined group after 3 and 6 months of therapy (28.6 versus $0 \%, \mathrm{P}=0.098$ and 35.7 versus $7.1 \%$, $\mathrm{P}=0.165$ ). 


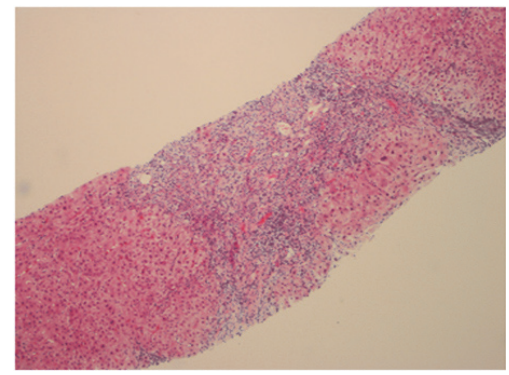

(a)

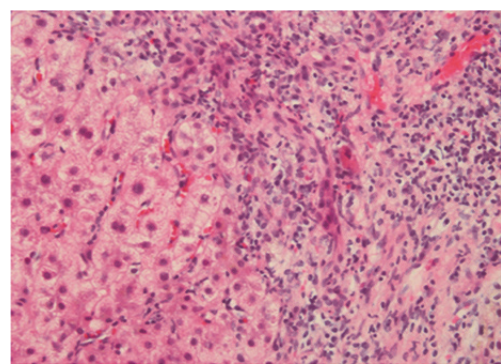

(c)

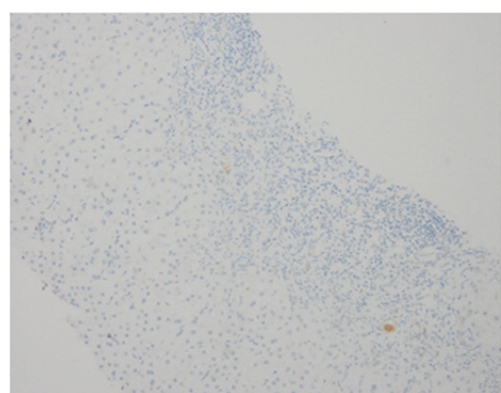

(e)

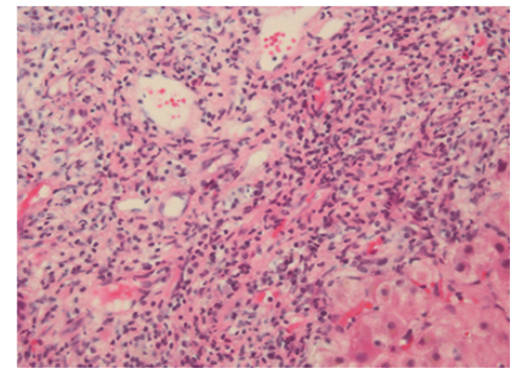

(b)

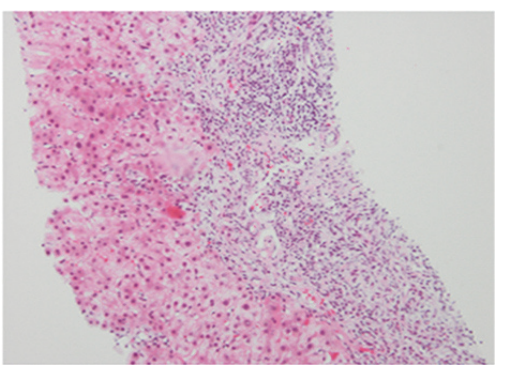

(d)

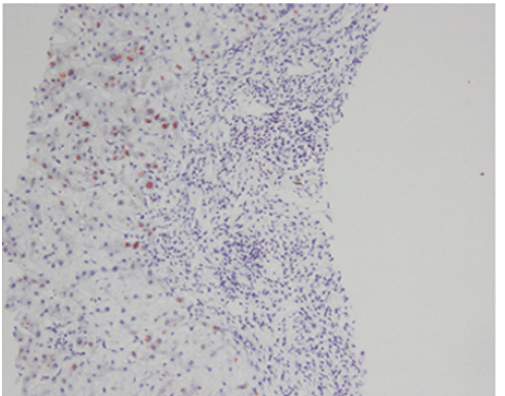

(f)

FIGURE 2: Histological features of decompensated PBC-AIH patients. (a) Bridging necrosis and moderate to severe interface hepatitis $(\times 100$, HE staining); (b) prominent interface hepatitis with numerous plasma cells ( $\times 400$, HE staining); (c) typical rosetting of hepatocytes in the area of interface hepatitis $(\times 400$, HE staining); (d), (e), (f) interlobular bile duct loss without a significant ductular reaction $(\times 200$, HE staining, CK7 staining, and copper staining in sequence).

TABLE 3: Histological features of the decompensated PBC-AIH patients.

\begin{tabular}{lccc}
\hline Variables & UDCA-only group (N=14) & UDCA+IS group (N=14) & P value \\
\hline Number of portal areas & 10 & 9 & 0.541 \\
Severe interface hepatitis & $0 / 14$ & $2 / 14$ & 0.481 \\
Moderate interface hepatitis & $14 / 14$ & $12 / 14$ & 0.481 \\
Hepatocyte rosette formation & $8 / 14$ & $10 / 14$ & 0.695 \\
Lymphoplasmacytic infiltrate & $13 / 14$ & $13 / 14$ & $>0.999$ \\
Emperipolesis & $0 / 14$ & $0 / 14$ & - \\
Bile duct damage & $13 / 14$ & $10 / 14$ & 0.326 \\
Ductopenia & $9 / 14$ & $6 / 14$ & 0.449 \\
Bile duct proliferation & $11 / 14$ & $9 / 14$ & 0.678 \\
Cholestasis & $7 / 14$ & $6 / 14$ & $>0.999$ \\
G0/1/2/3/4 (N) & $0 / 0 / 2 / 12 / 0$ & $0 / 1 / 2 / 7 / 4$ & 0.374 \\
S0/1/2/3/4 (N) & $0 / 3 / 4 / 5 / 2$ & $0 / 0 / 3 / 8 / 3$ & 0.117 \\
\hline
\end{tabular}

Note. UDCA, ursodeoxycholic acid; IS, immunosuppressants. 
TABLE 4: Response to treatment in AIH features after 3, 6, and 12 months of therapy.

\begin{tabular}{|c|c|c|c|c|}
\hline Variables & Overall $(\mathrm{n}=\mathbf{2 8}, \%)$ & UDCA-only group $(n=14), \%$ & UDCA+IS group $(n=14), \%$ & $P$ value \\
\hline 3-month remission & $4(14.2 \%)$ & $0(0 \%)$ & $4(28.6 \%)$ & 0.098 \\
\hline 6-month remission & $6(21.4 \%)$ & $1(7.1 \%)$ & $5(35.7 \%)$ & 0.165 \\
\hline 12 -month remission $*$ & $7(33.3 \%)^{\#}$ & $1(9.1 \%)^{\# \#}$ & $6(60.0 \%)$ & 0.024 \\
\hline
\end{tabular}

*Twenty-one patients were treated for more than 12 months in total.

\#Eleven patients were treated for more than 12 months in the UDCA-only group.

\#\# Ten patients were treated for more than 12 months in the UDCA+IS group.

TABLE 5: Liver-related adverse events in the two groups.

\begin{tabular}{lccc}
\hline Variables & UDCA-only group (N=14) & UDCA+IS group (N=14) & $\boldsymbol{P}$ value \\
\hline Adverse events & $9 / 14(64.3 \%)$ & $2 / 14(14.3 \%)$ & 0.018 \\
Severe ascites & $4(28.6 \%)$ & $1(7.1 \%)$ & 0.326 \\
Variceal bleeding & $1(7.1 \%)$ & 0 & $>0.999$ \\
Liver failure & $4(28.6 \%)$ & $1(7.1 \%)$ & 0.596 \\
Transplantation/liver-related death & $4 / 14(28.6 \%)$ & $1 / 14(7.1 \%)$ & 0.326 \\
\hline
\end{tabular}

Note. UDCA, ursodeoxycholic acid; IS, immunosuppressants.

The symptoms of $12 / 14(85.7 \%)$ of patients in the combined group have improved significantly, while the rate of symptom improvement was $71.4 \%(\mathrm{P}=0.353)$.

\subsection{Liver-Related Adverse Events and Transplant Free Survival} Period. Table 5 shows the clinical outcomes at the end of the study. During the study period, the rates of liver-related adverse events were $64.3 \%$ ( 9 of 14 ) and $14.3 \%$ ( 2 of 14 ) in the UDCA alone group and the combination group, respectively $(P=0.018)$. Thus, a total of 11 patients experienced liver-related adverse events during the follow-up. The median follow-up time was $18.0(13.3,20.8)$ months and was similar for patients in the UDCA-only group and the combined group [20.0 $(13.5,26.8)$ months, respectively $(\mathrm{P}=0.427]$. To date, relapses during maintenance therapy have not been observed, and immunosuppression has not been withdrawn.

Liver transplantations were more commonly observed in the UDCA-only group and the combined group (4/14 vs $1 / 14, \mathrm{P}=0.326)$. No patient died till the end of follow-up. The reasons for liver transplantation were cholestasis $(\mathrm{N}=3)$, esophagogastric hemorrhage $(\mathrm{N}=1)$, and refractory ascites $(\mathrm{N}=1)$. In the 5 patients who received liver transplantations in the two groups, none died posttransplantation. The KaplanMeier estimate showed that the transplant-free survival was distinct between the two groups $(\mathrm{P}=0.019)$ (Figure 3$)$.

3.5. Drug Side-Effects. Seven patients experienced transient diarrhoea after UDCA treatment. Corticosteroid-related side effects were noted in $35.7 \%$ (5 of 14) of the patients in the combined group. Most of these effects were mild, steroidspecific side effects, such as acne and moon face, and most resolved after dose reduction. Two patients in the UDCA+IS group experienced mild leukopenia (white blood count values of $3.0 \times 10^{9} / \mathrm{L}$ and $\left.2.49 \times 10^{9} / \mathrm{L}\right)$ during the first 60 days of AZA treatment. AZA was not discontinued, because the white blood counts increased gradually and thereafter

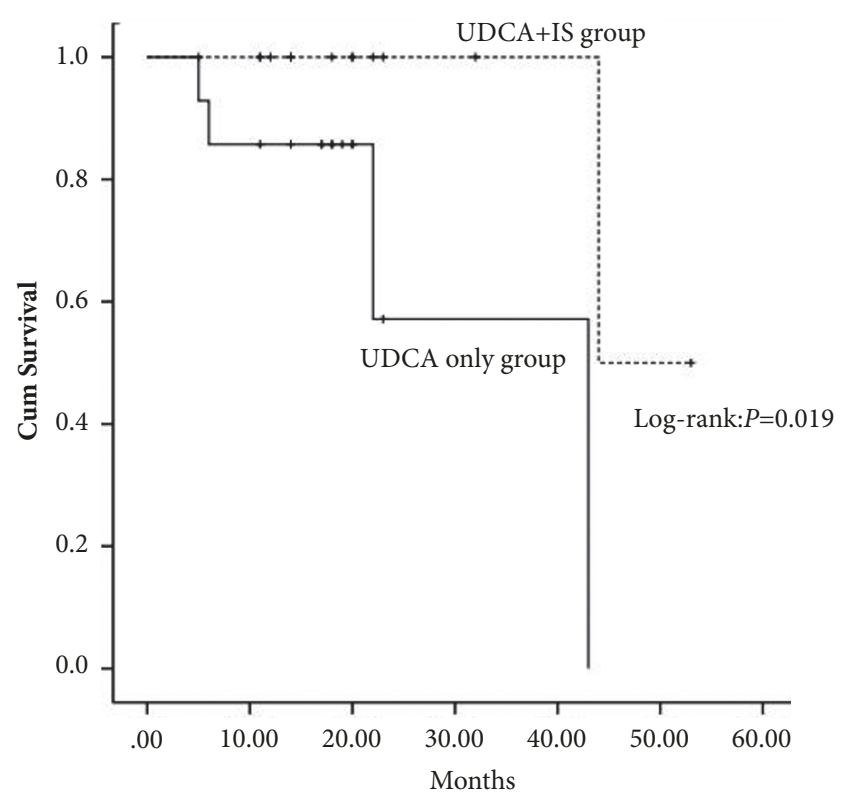

FIGURE 3: Transplant-free survival between the two groups (logrank, $\mathrm{P}=0.019$ ).

remained stable. A mild urinary tract infection occurred in one patient who received MMF during the first 60 days of AZA treatment.

\section{Discussion}

This cohort study was the first to assess the real-world effectiveness and safety of immunosuppressors in decompensated PBC-AIH patients. Six patients (60\%) receiving UDCA plus immunosuppressors achieved biochemical remission, whereas only one patient (9.1\%) achieved biochemical remission 12 months after beginning therapy. Patients treated with 
a combination of UDCA and immunosuppressors experienced fewer liver-related adverse events and obtained longer transplant-free survival, implying that the natural history and progression of decompensated PBC-AIH may also be haltered and averted.

Biochemical remission is a predictor of histologic outcome in PBC-AIH and may postpone the progression of cirrhosis [20]. In the present study, biochemical remission occurred in $60.0 \%$ of patients treated with a combination of UDCA and immunosuppression. Our data were consistent with the results from other studies, although these studies did not investigate advanced cirrhosis. A multicentre retrospective study with 88 patients with PBC-AIH found that the combination of UDCA and immunosuppression was effective in $73 \%$ of patients who were not previously treated or did not respond to UDCA alone [20]. Chazouilleres et al. found that fibrosis progression occurred more frequently in noncirrhotic patients under UDCA monotherapy (4/8) than under combined therapy $(0 / 6)(P=0.04)$ [21]. Similar results were obtained in a recent meta-analysis, which found that combination therapy with UDCA and corticosteroids was more effective than UDCA alone [6]. In our study, most of the biochemical and immune parameters improved dramatically in both groups after treatment, whereas the immune variable IgG, which is a hallmark of liver inflammation and the treatment response [5], did not decrease in the UDCA group. However, the TBIL values, which were used as one element to evaluate AIH remission [22], did not significantly differ before and after treatment in either group (data not shown). Early studies have suggested that UDCA alone can help achieve biochemical and histological improvements in PBC$\mathrm{AIH}$ patients, but the patient groups of the studies being compared did not focus on PBC-AIH overlap syndrome accompanied by decompensated cirrhosis [23, 24]. Hence, our data, similar to data from other studies, emphasize that the administration of immunosuppressors aids the hepatic immune response in this group of patients.

The median survival time for decompensated cirrhosis may be less than 2 years $[9,10]$. Recent EASL guidelines proposed that treatment was probably no longer indicated in AIH patients with decompensated cirrhosis unless they had a high inflammatory score on the liver biopsy. However, limited studies have offered real-world data for the responses, outcomes, and side effects in $\mathrm{AIH}$ or PBC-AIH patients with decompensated cirrhosis $[5,25]$. In the present study, the data showed a benefit on the progression of advanced cirrhosis in the UDCA+IS group during the observation period (median, 15.0 months). This result was in line with another study. Wang et al. found that immunosuppression treatment helped $62.5 \%$ of $\mathrm{AIH}$ patients with decompensated cirrhosis revert to compensated cirrhosis and that the rate of transplant-free survival was significantly greater in patients who received corticosteroids compared to those who did not [25]. Although the relationship between PBC-AIH overlap syndrome and $\mathrm{AIH}$ alone is complex, their results implied that immunosuppressive treatment could interrupt the progression of autoimmune-mediated advanced cirrhosis by controlling the hepatic immune response. In our study, all the patients were diagnosed according to Paris criteria; that is,
PBC patients were diagnosed with AIH when they met 2 or more of the criteria, which meant that our patients were with histological features of active liver inflammation, rather than quiet or burnt-out cirrhosis. In that situation, more emphasis would be needed on the treat complications to prolong the transplant-free survival. Hence, individualized protocols are needed for different clinical situations. However, further prospective studies with more patients and long-term followups may provide more robust evidence.

Prior to starting therapy, the risks and benefits of immunosuppressive treatment must be weighed. In our study, 12 patients $(85.7 \%)$ received an initial methylprednisolone dose of $24 \mathrm{mg} / \mathrm{d}$ that was rapidly tapered according to the treatment response. The methylprednisolone doses for the other two patients were $12 \mathrm{mg} / \mathrm{d}$ and $48 \mathrm{mg} / \mathrm{d}$. No serious sideeffects of methylprednisolone were observed in our study, which was mostly attributed to our positive strategies used for tapering, preventive medications, and monitoring. In the 12 patients who received AZA as an adjuvant drug, transient leukopenia occurred in 2 patients (14.2\%). Our data were not consistent with Heneghan et al. [26], who found that advanced fibrosis but not the thiopurine methyltransferase (TPMT) genotype or activity predicted azathioprine toxicity in AIH patients. In our patients, NUDT15 (i.e., rs116855232) and TPMT (i.e., rs1142345) SNPs were genotyped using a realtime PCR method. No TPMT genetic variants were genotyped in these 12 patients, but heterozygous NUDT15 R139C genotypes were found in the 2 patients who experienced leukopenia (data not shown). Hence, adjusting the azathioprine dosage may be considered according to the rs116855232 genotype rather than advanced fibrosis or cirrhosis. Hence, the rate of leukopenia was relatively low and acceptable. However, whether the TPMT and NUDT genotypes were associated with leukopenia in these $\mathrm{PBC}-\mathrm{AIH}$ patients is unknown. Also, the infection rate was $7.1 \%$, which was in line with Wang et al. (10.9\%) [25].

The present study has some limitations. First, this investigation was a single-centre cohort study. Given this experimental approach, avoiding confounding factors was relatively difficult, and some data were not available. Second, because decompensated cirrhosis is an uncommon and advanced presentation of this disease, obtaining larger sample sizes is difficult in real-word studies. The current lack of controlled trials makes the results of small studies on rare diseases helpful and informative. Furthermore, because the mortality rate is as high as $85 \%$ over 5 years in patients with decompensation who do not receive a liver transplant, conducting a prospective study with extended follow-up can be very difficult. Third, no histological evaluations were performed after therapy to confirm the histological validity of this study.

In conclusion, we found that PBC-AIH patients with decompensated cirrhosis receiving a combination of UDCA and immunosuppressors presented higher biochemical remission rates, fewer liver-related adverse events, and longer transplant-free survival, implying that combined treatment might be a better therapeutic option for strictly defined PBC-AIH overlap syndrome accompanied by decompensated cirrhosis. 


\section{Data Availability}

All data arising from this study are contained within the manuscript.

\section{Ethical Approval}

The study was reviewed and approved by the Ethics Committee of the West China Hospital, Sichuan University (no. 2013221).

\section{Consent}

All subjects provided written informed consent before enrolment.

\section{Conflicts of Interest}

The authors declare that there are no conflicts of interest regarding the publication of this article.

\section{Authors' Contributions}

Xiaoli Fan and Yongjun Zhu contributed equally to this work.

\section{Acknowledgments}

This work was supported by grants from the National Natural Science Foundation of China [no. 81770568 to Li Yang] and Fundamental Research Funds for the Central Universities [no. 2012017yjsy198 to Xiaoli Fan].

\section{References}

[1] A. J. Czaja, "Frequency and nature of the variant syndromes of autoimmune liver disease," Hepatology, vol. 28, no. 2, pp. 360365, 1998.

[2] C. Rust and U. H. Beuers, "Overlap syndromes among autoimmune liver diseases," World Journal of Gastroenterology, vol. 14, no. 21, pp. 3368-3373, 2008.

[3] O. Chazouillères, D. Wendum, L. Serfaty, S. Montembault, O. Rosmorduc, and R. Poupon, "Primary biliary cirrhosisautoimmune hepatitis overlap syndrome: clinical features and response to therapy," Hepatology, vol. 28, no. 2, pp. 296-301, 1998.

[4] A. Heurgué, F. Vitry, M.-D. Diebold et al., "Overlap syndrome of primary biliary cirrhosis and autoimmune hepatitis: A retrospective study of 115 cases of autoimmune liver disease," Gastroentérologie Clinique et Biologique, vol. 31, no. 1, pp. 17-25, 2007.

[5] "Corrigendum to "EASL Clinical Practice Guidelines: Autoimmune hepatitis" [J Hepatol 2015;63:971-1004]," Journal of Hepatology, vol. 63, no. 6, pp. 1543-1544, 2015.

[6] H. Zhang, S. Li, J. Yang et al., "A meta-analysis of ursodeoxycholic acid therapy versus combination therapy with corticosteroids for PBC-AIH-overlap syndrome: Evidence from 97 monotherapy and 117 combinations," Przegląd Gastroenterologiczny, vol. 10, no. 3, pp. 148-155, 2015.

[7] G. M. Hirschfield, U. Beuers, C. Corpechot et al., "EASL Clinical Practice Guidelines: The diagnosis and management of patients with primary biliary cholangitis," Journal of Hepatology, vol. 67, no. 1, pp. 145-172, 2017.

[8] G. Garcia-Tsao, J. G. Abraldes, A. Berzigotti, and J. Bosch, "Portal hypertensive bleeding in cirrhosis: risk stratification, diagnosis, and management: 2016 practice guidance by the American Association for the study of liver diseases," Hepatology, vol. 65, no. 1, pp. G310-G335, 2017.

[9] A. Zipprich, G. Garcia-Tsao, S. Rogowski, W. E. Fleig, T. Seufferlein, and M. M. Dollinger, "Prognostic indicators of survival in patients with compensated and decompensated cirrhosis," Liver International, vol. 32, no. 9, pp. 1407-1414, 2012.

[10] G. D’Amico, L. Pasta, A. Morabito et al., "Competing risks and prognostic stages of cirrhosis: A 25-year inception cohort study of 494 patients," Alimentary Pharmacology \& Therapeutics, vol. 39, no. 10, pp. 1180-1193, 2014.

[11] A. W. Lohse and G. Mieli-Vergani, "Autoimmune hepatitis," Journal of Hepatology, vol. 55, no. 1, pp. 171-182, 2011.

[12] K. M. Boberg, R. W. Chapman, G. M. Hirschfield, A. W. Lohse, M. P. Manns, and E. Schrumpf, "Overlap syndromes: the International Autoimmune Hepatitis Group (IAIHG) position statement on a controversial issue," Journal of Hepatology, vol. 54, no. 2, pp. 374-385, 2011.

[13] P. J. Trivedi and G. M. Hirschfield, "Review article: Overlap syndromes and autoimmune liver disease," Alimentary Pharmacology \& Therapeutics, vol. 36, no. 6, pp. 517-533, 2012.

[14] R. T. Stravitz, J. H. Lefkowitch, R. J. Fontana et al., "Autoimmune acute liver failure: proposed clinical and histological criteria," Hepatology, vol. 53, no. 2, pp. 517-526, 2011.

[15] D. Schuppan and N. H. Afdhal, "Liver cirrhosis," The Lancet, vol. 371, no. 9615, pp. 838-851, 2008.

[16] P. J. Scheuer, "Classification of chronic viral hepatitis: a need for reassessment," Journal of Hepatology, vol. 13, no. 3, pp. 372-374, 1991.

[17] C. Sempoux and J. Rahier, "Histological scoring of chronic hepatitis," Acta Gastro-Enterologica Belgica, vol. 67, no. 3, pp. 290-293, 2004.

[18] A. W. H. Chan, R. C. K. Chan, G. L. H. Wong et al., "Evaluation of histological staging systems for primary biliary cirrhosis: Correlation with clinical and biochemical factors and significance of pathological parameters in prognostication," Histopathology, vol. 65, no. 2, pp. 174-186, 2014.

[19] T. Namisaki, K. Moriya, M. Kitade et al., "Clinical significance of the Scheuer histological staging system for primary biliary cholangitis in Japanese patients," European Journal of Gastroenterology \& Hepatology, vol. 29, no. 1, pp. 23-30, 2017.

[20] E. Ozaslan, C. Efe, A. Heurgué-Berlot et al., "Factors associated with response to therapy and outcome of patients with primary biliary cirrhosis with features of autoimmune hepatitis," Clinical Gastroenterology and Hepatology, vol. 12, no. 5, pp. 863-869, 2014.

[21] O. Chazouillères, D. Wendum, L. Serfaty, O. Rosmorduc, and R. Poupon, "Long term outcome and response to therapy of primary biliary cirrhosis - Autoimmune hepatitis overlap syndrome," Journal of Hepatology, vol. 44, no. 2, pp. 400-406, 2006.

[22] M. P. Manns, A. J. Czaja, J. D. Gorham et al., "Diagnosis and management of autoimmune hepatitis," Hepatology, vol. 51, no. 6, pp. 2193-2213, 2010.

[23] E. J. Heathcote, K. Cauch-Dudek, S. Joshi et al., "Primary biliary cirrhosis with additional features of autoimmune hepatitis: Response to therapy with ursodeoxycholic acid," Hepatology, vol. 35 , no. 2 , pp. 409-413, 2002. 
[24] F. Günsar, U. S. Akarca, G. Ersöz, Z. Karasu, G. Yüce, and Y. Batur, "Clinical and biochemical features and therapy responses in primary biliary cirrhosis and primary biliary cirrhosis-autoimmune hepatitis overlap syndrome," HepatoGastroenterology, vol. 49, no. 47, pp. 1195-1200, 2002.

[25] Z. Wang, L. Sheng, and Y. Yang, "he Management of Autoimmune Hepatitis Patients with Decompensated Cirrhosis: RealWorld Experience and a Comprehensive Review," Clinical reviews in allergy \& immunology, vol. 52, no. 3, pp. 424-435, 2016.

[26] M. A. Heneghan, M. L. Allan, J. D. Bornstein, A. J. Muir, and D. A. Tendler, "Utility of thiopurine methyltransferase genotyping and phenotyping, and measurement of azathioprine metabolites in the management of patients with autoimmune hepatitis," Journal of Hepatology, vol. 45, no. 4, pp. 584-591, 2006. 


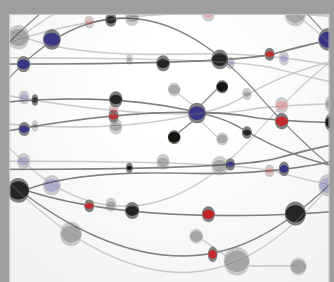

The Scientific World Journal
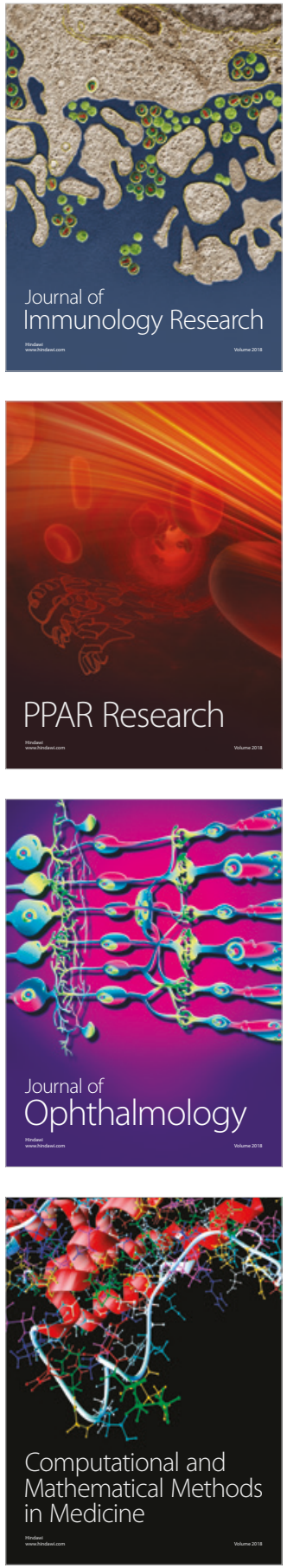

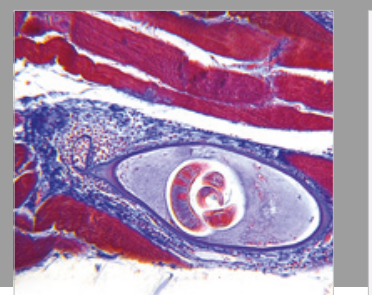

Gastroenterology Research and Practice

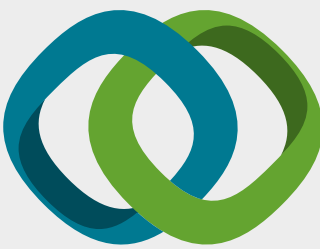

\section{Hindawi}

Submit your manuscripts at

www.hindawi.com
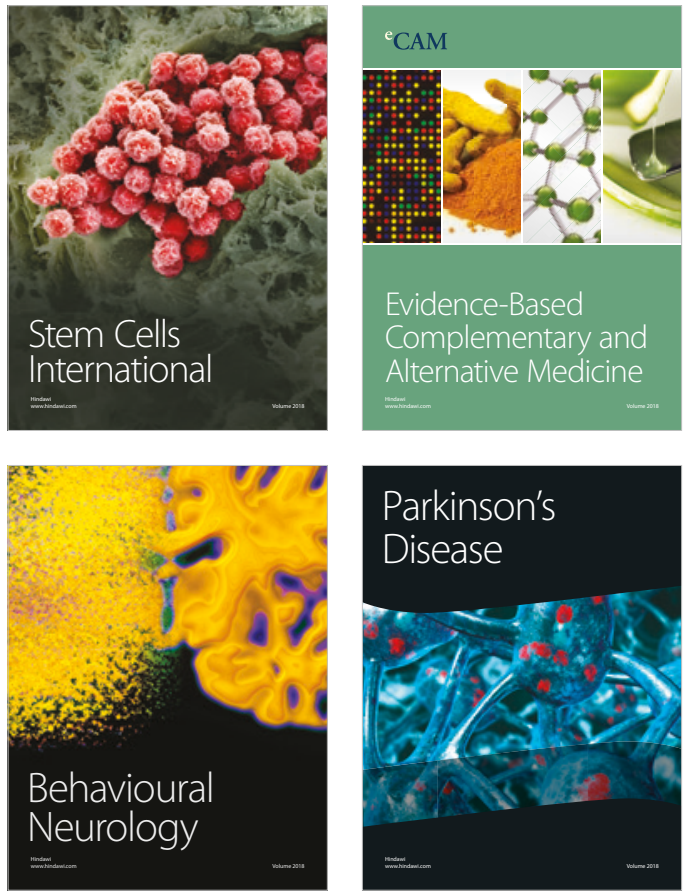

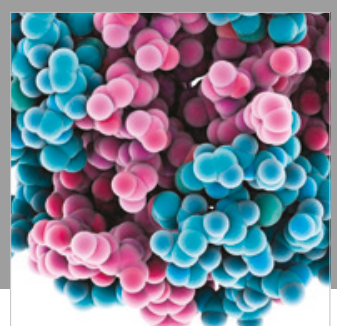

ournal of

Diabetes Research

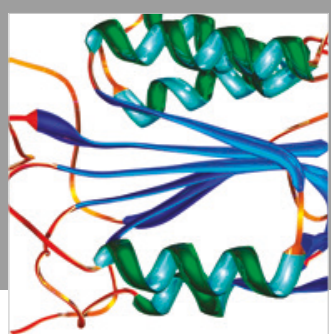

Disease Markers
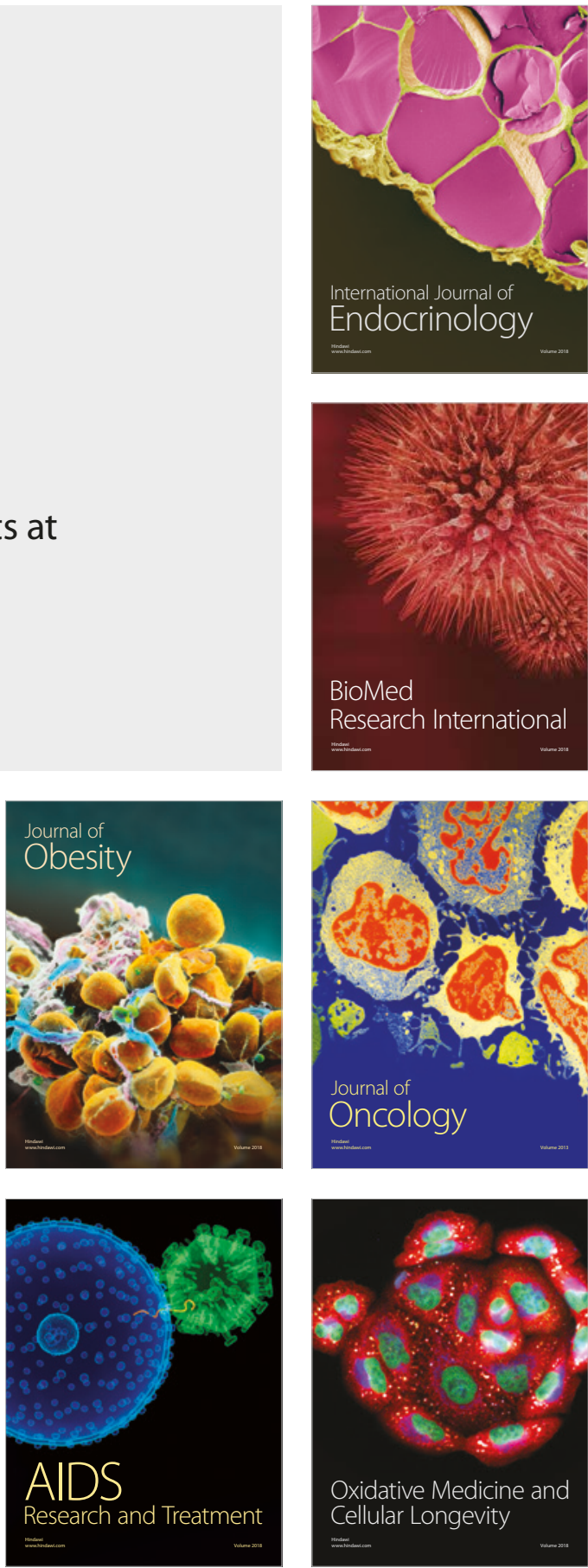